\title{
Variables ligadas al tabaquismo en una muestra de estudiantes de Ciencias de la Salud de la UDC
}

Variables Linked to Smoking in a Sample of Students of Health Sciences of the UDC

\section{Manuela Blanco Presas}

Unidad de Salud Mental Infanto-Juvenil Hospital Universitario A Coruña. A Coruña, España.

Escuela Universitaria Enfermería A Coruña. A Coruña, España.

Contacto: manuela.blanco.presas@sergas.es

Fecha de recepción: 22 de junio de 2018 / Fecha de aceptación: 19 de octubre de 2018

\begin{abstract}
Resumen
Introducción: El tabaquismo constituye un problema de salud pública sobre el que es necesario actuar para disminuir la morbilidad y mortalidad asociadas al mismo, siendo necesaria la toma de conciencia de los profesionales de salud, los cuales constituyen modelos sociales, con una gran capacidad de influencia en materia preventiva, por lo cual sería deseable erradicar dicha dependencia en los mismos. La estrategia de búsqueda bibliográfica se dirigió a encontrar artículos relevantes en los buscadores: Pubmed, biblioteca Cochrane, Fisterrra.com)

No existen estudios previos en muestras de estudiantes de Ciencias de la salud en la Universidad de A Coruña.

Metodología: Estudio descriptivo observacional de prevalencia en una muestra de 82 estudiantes de $1^{\circ}$ de Enfermería de A Coruña y de $3^{\circ}$ de Podología de Ferrol pertenecientes al curso 2009.

Resultados: Existe un predominio de mujeres fumadoras, como corresponde al género predominante en el alumnado de los centros académicos estudiados. La prevalencia del tabaquismo es del $37.8 \%$. La mayoría (79.3\%) fuma menos de diez cigarrillos al día.
\end{abstract}

Palabras clave: tabaquismo, deshabituación tabáquica, cooximetría.

\begin{abstract}
Introduction: Smoking is a public health problem on which it is necessary to act to reduce the morbidity and mortality associated with it, being necessary the awareness of health professionals, who are social models, with a great capacity for influence in preventive matter, for which it would be desirable to eradicate this habit in them.

The bibliographic search strategy was directed to find relevant articles in the search engines: Pubmed, Cochrane library, Fisterrra.com

There are no previous studies in samples of students of Health Sciences at the University of A Coruña.

Methodology: Observational descriptive study of prevalence in a sample of 82 students of 1st Nursing of A Coruña and 3rd Podology of Ferrol belonging to the 2009 course.

Results: There is a predominance of smoking women, as corresponds to the predominant gender in the students of the academic centers studied. The prevalence of smoking is $37.8 \%$. The majority $(79.3 \%)$ smoke less than ten cigarettes a day.
\end{abstract}

Keywords: smoking, smoking cessation, co-oximetry.

\section{Introducción}

El tabaquismo continúa siendo un grave problema de salud pública, que provoca altas tasas de morbilidad y supone en los países desarrollados la principal causa prevenible de enfermedad y muerte prematura ${ }^{1}$.
Los profesionales de la salud conforman el colectivo social con mayor influencia en la deshabituación tabáquica $^{2}$, a pesar de lo cual todavía existe una alta prevalencia de fumadores en profesionales de la salud, lo cual justifica este estudio debido a las implicaciones que se puedan derivar de la información obtenida ${ }^{3,4}$. 
Además de realizar un esfuerzo por conocer datos sobre la prevalencia de tabaquismo en este colectivo de estudiantes de la Universidad de A Coruña (UDC), es oportuno concretar las variables que influyen en la dependencia y la motivación para dejar de fumar, así como los motivos por los que se fuma ${ }^{5,6}$.

Se ha demostrado la gran utilidad del consejo breve para deshabituación tabáquica realizado por profesionales de la salud ${ }^{7-11}$.

\section{Metodología}

El ámbito de dicho estudio fue la Escuela de Enfermería y Podología de la Universidad de A Coruña (España). La población de estudio fueron estudiantes de $1^{\circ}$ de Enfermería de A Coruña y de $3^{\circ}$ de Podología de Ferrol durante el curso 2009. El tipo de estudio fue observacional de prevalencia, con los siguientes criterios de inclusión:

- Participación voluntaria.

- Ser estudiante durante el período de estudio de $1^{\circ}$ de Enfermería en la Escuela Universitaria de ACoruña.

- Ser estudiante de $3^{\circ}$ de Podología de la UDC durante el período de estudio.

Como criterios de exclusión se utilizaron:

- No desear participar en el estudio.

- No ser estudiante durante el período de estudio de $1^{\circ}$ de Enfermería en la Escuela Universitaria de A Coruña.

- No ser estudiante de $3^{\circ}$ de Podología de la UDC durante el período de estudio.

Se tuvieron en cuenta las consideraciones ético-legales referidas a:

- Consentimiento informado del alumno.

- Autorización de los responsables académicos de cada centro.

- Garantía de confidencialidad según la ley de protección de datos de carácter personal.

- Autorización del comité ético de investigación clínica de Galicia.

En cuanto al tamaño muestral, señalar que el tamaño actual de 82 alumnos nos permite estimar las características generales de la muestra estudiada en relación a la prevalencia del tabaquismo y hallazgos de cooximetría con una seguridad del $95 \%(\alpha=0.05)$ y una preci- sión de $\pm 11 \%$.

El número de fumadores en este estudio es de 31. Dicho tamaño muestral en relación a los cuestionarios utilizados en este subgrupo nos permite estimar los parámetros de interés con una seguridad del $95 \%(\alpha=$ $0.05)$ y una precisión de $\pm 18 \%$.

Con respecto a las mediciones realizadas, de cada alumno participante se estudiaron las siguientes variables:

\section{Variables de identificación:}

Edad, sexo, curso, facultad...

\section{Variables de consumo de tabaco:}

Hábito tabáquico, edad de inicio del consumo, edad de cese del consumo, número de cigarrillos actuales ( 0 10 y 11 a 20$)$.

Variables de exposición al tabaco medidas por cooximetría:

Cooximetría (p.p.m., carboxiHB, fcoHB). Permite conocer la cantidad de Monóxido de Carbono (CO) en aire espirado y su equivalencia en Carboxihemoglobina $(\mathrm{COHb})$. Sólo valora consumos recientes(24-48 horas). Las cifras de $\mathrm{CO}$ se sitúan generalmente en:

- por debajo de 5 partes por millón (p.p.m.) en personas no fumadoras;

- entre 5-10 p.p.m. en fumadores ocasionales;

- entre 5-10 p.p.m. en fumadores ocasionales o fumadores que realizan inhalaciones muy superficiales;

- por encima de 10 p.p.m. en fumadores diarios.

\section{Cuestionarios utilizados:}

a) Test de Richmond: mide la motivación para dejar de fumar. Consta de cuatro preguntas y cada ítem varía en una escala de Likert de 0 a 3 , excepto el primero, en el que se puede obtener 0 o 1 punto. Se puede obtener una puntuación máxima de diez puntos, que nos permite clasificar la motivación para dejar de fumar en débil, de 0 a 5 puntos, media, de 6 a 8 puntos, o fuerte, si se obtienen 9 o más puntos.

b) Test de Fagerström: cuantifica la dependencia a la nicotina, tiene 6 preguntas y su puntuación oscila entre 0 y 10 , reflejando el valor 10 la mayor dependencia. 
c) Test de Russell: evalúa los diferentes motivos por los que se fuma: imagen psicológica, actividad bocamano, indulgencia, sedación, automatismo, adicción, dependencia global, estimulación. Consta de 24 ítems en una escala de Likert, con las categorías: 0 , no le sucede en absoluto; 1 , le sucede un poco; 2 , le sucede bastante; 3, le sucede mucho. La mayor puntuación expresa más motivos para seguir fumando. La máxima puntuación es de 72 puntos, y la mínima de 0. Cada motivo alcanza como máximo una puntuación de 9 puntos, siendo considerado relevante si posee una puntuación mayor de 6 puntos.

d) Test de Baer \& Lichtenstein: analiza el grado de resistencia para no fumar ante determinadas situaciones. Presenta 14 ítems en una escala de Likert, que va de 0 (no fumaría) a 10 (sí fumaría). La máxima puntuación es de 140 puntos y la mínima de 0 .

La mayor puntuación expresa menor resistencia que opondría cada alumno al deseo de fumar en determinadas situaciones.

e) Test de Glover-Nilsson: analiza el grado de dependencia psicológica, social y gestual que una persona posee frente al tabaco. Consta de 11 ítems y tiene una puntuación entre 0 y 44. De nuevo, una mayor puntuación refleja una mayor dependencia al tabaco. Cada ítem consta de cuatro posibles respuestas que puntúan desde 0 al 4; nunca (0), raramente, a veces, muy a menudo, siempre (4). Este cuestionario clasifica la dependencia psicológica, según la puntuación obtenida, en leve $(<12)$, moderada $(12-22)$, fuerte (23-33) y muy fuerte ( $>33)$. De nuevo, como en los test anteriores, la mayor puntuación supone una mayor dependencia al tabaco.

Análisis estadístico: se realizó un estudio descriptivo de las variables incluidas en el estudio. Las variables cuantitativas se expresan como media \pm desviación típica. Las variables cualitativas como valor absoluto y porcentaje. La comparación de medias se realizó con la T de Student o con el test de Mann-Whitney según procediese, tras comprobación de la normalidad con el test de Kolmogorov-Smirnov. La comparación múltiple se realizó con el estadístico de Kruskal-Wallis. La correlación entre diferentes variables cuantitativas se realizó por medio del coeficiente de correlación de Pearson cuando las variables seguían una distribución normal y el coeficiente de Spearman en el caso de las que no seguían una distribución normal.

\section{Resultados}

Las características de la muestra estudiada se muestran en la tabla 1.

Predominan las mujeres fumadoras como corresponde a los centros académicos estudiados. La prevalencia del tabaquismo es del $37.8 \%$. La mayoría (79.3\%) fuma menos de diez cigarrillos al día.

Los hallazgos de la cooximetría realizada se muestran en la tabla 2 y figuras 1-3.

La media de los valores de las mediciones de la cooximetría según el hábito tabáquico declarado se muestran en la tabla 3 , objetivándose valores más elevados en fumadores actuales, siendo las diferencias estadísticamente significativas.

\section{Resultados de los cuestionarios}

\section{Cuestionario de Richmond.}

Los resultados de dicho cuestionario y la puntuación global se muestran en la tabla 4 y figura 4.

Objetivándose que al 86.2\% les gustaría dejar de fumar si lo pudieran hacer fácilmente, aunque el $62.1 \%$ no están dispuestos a dejar de fumar en las próximas dos semanas.

En nuestro estudio (gráfica 4), la media es de $4.52 \pm$ 2.52. En dicho histograma se objetivan 2 grupos de alumnos: los más motivados y los menos motivados, estando la mediana en 4.

Aunque los hombres están más motivados para dejar de fumar que las mujeres, la diferencia no es estadísticamente significativa $(5.3 \pm 3.1$ vs. $4.3 \pm 2.4)$. Igualmente sucede en la comparación de centros; los alumnos de podología están más motivados a dejar de fumar que los alumnos de enfermería aunque la diferencia tampoco es significativa $(5.1 \pm 2.2$ vs. $3.9 \pm$ 2.8).

Existe una correlación muy débil entre la edad de inicio y la motivación para dejar de fumar, que no es estadísticamente significativa $(r=0.19 ; \mathrm{p}=0.37)$. La correlación entre la edad actual y la motivación para dejar de fumar es incluso aún más débil que con la edad de inicio y tampoco es significativa $(r=0.089$; $\mathrm{p}=0.65)$. 
Los valores de la cooximetría tampoco se relacionan con la motivación para dejar de fumar, siendo los valores de los coeficientes de correlación próximos a cero (ppp, r = 0.09; carboxiHb $=0.07)($ tabla 9).

\section{Cuestionario de Fagerström.}

Este cuestionario cuantifica la dependencia a la nicotina $^{12,13}$. Los resultados se muestran en la tabla 5 y figura 5 .

La mayoría de los alumnos fumadores (86.2\%) consume diez o menos cigarrillos al día, y también la mayoría de los fumadores $(75.9 \%)$ fuma el primer cigarrillo a los 5 minutos o menos de despertarse. El $79.3 \%$ tiene dificultades para abstenerse de fumar en los lugares donde está prohibido hacerlo, y el cigarrillo que más se necesita a lo largo del día es el primero de la mañana $(89.7 \%)$.

La media del cuestionario de Fagerström está en $1.17 \pm 1.87$ con un rango que oscila entre 0 y 8 (Figura 5).

Aunque los hombres tienen una puntuación de dependencia a la nicotina discretamente mayor que las mujeres, la diferencia no es significativa $(1.33 \pm 1.7$ vs. $1.13 \pm 1.9)(p=0.73)$. A su vez objetivamos que los alumnos de podología tienen mayor dependencia que los alumnos de enfermería, no siendo significativa la diferencia $(1.4 \pm 2.32$ vs. $0.93 \pm 1.26)(\mathrm{p}=0.98)$.

Existe una débil correlación positiva entre la puntuación del test de Fagerström y la edad de inicio $(r=0.14)$ y la edad actual $(r=0.064)$, que no es estadísticamente significativa.

El test de Fagerström se correlaciona con los valores de la cooximetría ${ }^{14}$ siendo el coeficiente de correlación de Spearman más elevado para la CarboxiHB (0.33) estando dicha correlación en el límite de ser significativa (0.09). A mayor dependencia mayor valor de CarboxiHB ${ }^{15-16}$ (tabla 9).

\section{Cuestionario de Russell.}

Este cuestionario analiza los diferentes motivos por los que se fuma ${ }^{17}$.

Los resultados (tabla 6) muestran una media de $17.6 \pm 10.7$, con un rango que oscila entre 0 y 50 y una mediana de 19 puntos.
El ítem que tiene la puntuación más elevada $(41,4 \%)$ (le sucede mucho) es el de "Fumo más cuando estoy preocupado por algo"; le sigue, con el $24.1 \%$, "Me gusta fumar, sobre todo cuando descanso tranquilamente", y en tercer lugar $(20.7 \%)$ "Me resultaría muy difícil estar una semana sin fumar".

Se objetiva que no existe una diferencia significativa $(p=0.97)$ en cuanto al centro de estudio; la puntuación media de los estudiantes de enfermería es de 17.5 \pm 9.93 y los de podología tienen una puntuación media de $17.67 \pm 11.79$, de lo que se deduce que los futuros podólogos y enfermeros tienen los mismos motivos para fumar.

En cuanto al género, se observa que existe una diferencia significativa $(\mathrm{p}=0.01)$ entre ser mujer o hombre $(20.09 \pm 10.29$ vs. $8 \pm 6.4)$. Es decir, las mujeres encuentran más motivos por los que seguir fumando.

Existe una correlación débil entre la edad $(\mathrm{r}=-0.41$; $p=0.03$ ) y los diferentes motivos por los que se fuma. Se observa que a mayor puntuación en el test de Russell, menor edad del alumno ${ }^{18-19}$, lo que se traduce en que cuanto menor es la edad, existen más motivos para fumar (figura 7). En Galicia fuman a diario un 19.3\% de la población mayor de 16 años; otro $2.1 \%$ lo hacen de forma ocasional.

La correlación entre la edad de inicio y los motivos por los que se fuma es muy débil y no es estadísticamente significativa $(r=-0.129 ; p=0.54)$.

Los valores de la cooximetría tampoco se relacionan con los motivos por los que se fuma, aunque con la carboxiHb existe una correlación muy débil que está a punto de ser significativa $(r=0.324 ; p=0.093)$ (tabla 9).

\section{Cuestionario de Baer \& Lichtenstein.}

Este cuestionario analiza el grado de resistencia para no fumar ante determinadas situaciones (tabla 7).

La media de dicho cuestionario es de $63.38 \pm 27.33$ con un rango que oscila entre 0 y 104 y una mediana de 69 puntos.

La mayoría de los alumnos (75.9\%) no fumarían en las siguientes situaciones: "Si quisiera sentirme más atractivo", "Si quisiera sentirme más maduro y sofisticado", "Si pensara que fumar es parte de mi auto- 
imagen", todas ellas claramente relacionadas con la autoimagen $^{20}$; le siguen con un porcentaje bastante elevado los ítems "Si pensara que esto me ayudaría a mantener mi peso" y "Si quisiera evitar comer algo dulce", en los cuales la mayoría de los alumnos, $51.7 \%$ y $44.8 \%$ respectivamente, también puntúan cero.

En contraposición, el ítem en el que gran parte de los alumnos (34.5\%) no se resistiría a fumar (puntúan 10) es "Si estuviera bebiendo una bebida alcohólica", lo que nos remite a la alianza alcohol como facilitador del consumo de tabaco ${ }^{21,22}$.

De los 29 encuestados (23 son mujeres y 6 son hombres), se observa que las mujeres tienen de media una mayor puntuación que los hombres $(69.91 \pm 5.23$ vs. $38.33 \pm 21.53(p=0.009)$; es decir, las mujeres ofrecen de media una menor resistencia a fumar. Sin embargo, no se observa una diferencia significativa $(p=0.97)$ entre el grado de resistencia a no fumar entre futuros enfermeros y podólogos; la puntuación media es de $63.57 \pm 28.43$ y de $63.2 \pm 27.26$ puntos respectivamente.

En este caso, no se objetiva ninguna correlación entre la edad actual del alumno, la edad de inicio ni con los diferentes valores de la cooximetría (tabla 9).

\section{Cuestionario de Glover-Nilsson.}

El test de Glover-Nilsson analiza el grado de dependencia psicológica, social y gestual que una persona posee frente al tabaco (tabla 11). Las puntuaciones obtenidas oscilan entre 0 y 32 puntos, alcanzándose la media en los $11.14 \pm 7.42$ puntos. Se observa que el $62.1 \%$ de los alumnos tiene una adicción leve al tabaco, el $31 \%$ tienen una adicción moderada y el resto $(6.9 \%)$ de los alumnos tienen una adicción fuerte, no observándose ningún caso de adicción muy fuerte.

La mayoría de los alumnos (82.8\%) nunca coloca cigarrillos en los labios sin encender u otros objetos similares para evitar fumar. En contraposición, el $62 \%$ de los encuestados reconoce que muy a menudo o siempre algunos lugares o circunstancias les incitan a fumar.

Podemos afirmar que existe una diferencia significativa $(p=0.04)$ entre la dependencia gestual psicológica y social de las mujeres y los hombres (12.57 \pm 7.4 ; $5.67 \pm 4.72)$, indicando una dependencia leve en hom- bres, moderada en mujeres. Observamos mayor dependencia al tabaco en alumnos de enfermería con respecto a los de podología $(12.5 \pm 7.1$ vs. $9.87 \pm$ 7.74); la diferencia en este caso tampoco es estadísticamente significativa $\mathrm{p}=0.35$.

Existe una correlación muy débil entre la edad de inicio y la dependencia gestual, psicológica y social al tabaco, que no es estadísticamente significativa $(r=-0.14 ; p=0.52)$. Sin embargo, existe una correlación significativa entre la edad actual y la dependencia estudiada $(r=-0.39 ; p=0.04)$.

Los valores de la cooximetría tampoco se relacionan significativamente con la motivación para dejar de fumar, siendo los valores de los coeficientes de correlación próximos a cero en el caso de fcoHB $(r=0.02)$ y un poco mayores en el caso de p.p.m. y carboxiHB $(r=0.19 ; r=0.22$, respectivamente $)$.

A continuación se analizará la relación existente entre los diferentes test utilizados ${ }^{23-25}$. Se objetiva (tablas 8 y 10) que existe una correlación significativa, aunque débil, entre el cuestionario de Russell y el de Richmond ( $\mathrm{r}=-0.42 ; \mathrm{p}=0.03)$, lo que supone que, a mayor puntuación en el cuestionario de Russell, menor puntuación en el test de Richmond. Es decir, cuantos más motivos se tengan para fumar, menos motivos se tendrán para dejar de fumar.

También se observa una correlación significativa entre el cuestionario de Richmond y el de Baer \& Lichtenstein $(\mathrm{r}=-0.59 ; \mathrm{p}=0.001)$; esto se traduce en que una menor resistencia a no fumar en determinadas situaciones supone menor motivación para dejar de fumar.

De la misma manera existe una correlación entre el cuestionario de Russell y el de Baer \& Lichtenstein $(r=0.79 ; p=0.00)$, de lo que se deduce que cuanto más motivos por los que se fuma, menor será la resistencia a no fumar.

Se objetiva una correlación negativa y significativa entre los cuestionarios de Glover-Nilsson y Richmond, $(r=-0.45 ; p=0.01)$; esto supone que una mayor dependencia psicológica, social y gestual implica una menor motivación para dejar de fumar.

Igualmente existe una fuerte correlación entre el cuestionario de Glover-Nilsson y Russell $(r=0.85$; $\mathrm{p}=0.00)$, lo que se traduce en que a mayor dependen- 
cia psicológica, social y gestual, existen más motivos por los que fumar.

Finalmente, existe una correlación significativa entre el cuestionario de Glover-Nilsson y el de Baer \& Lichtenstein $(r=0.72 ; p=0.00)$; esto indica que a mayor dependencia social, psicológica y gestual, menor resistencia a no fumar ante determinadas situaciones.

El cuestionario de Fagerström es el único que no correlaciona con ningún otro de los cuestionarios utilizados en este estudio (tabla 8).

\section{Discusión}

A pesar de las recomendaciones de la OMS en materia de deshabituación tabáquica, muchos profesionales de la salud siguen manteniendo su consumo ${ }^{1,45-7}$, y aun reconociéndolo como un problema de salud pública de primera magnitud $^{11}$, su consumo no está erradicado entre dichos profesionales ${ }^{5,15,27}$.

Parece oportuno concienciar a los estudiantes de Ciencias de la Salud a desarrollar conductas saludables coherentes con el modelo social que están destinados a desarrollar ${ }^{2,15}$. En España la prevalencia del tabaquismo es del $31 \%$ en la población adulta, y aunque se observa una disminución en la prevalencia ${ }^{21,23,27}$, a lo cual han contribuido fundamentalmente las medidas legislativas reguladoras del consumo de tabaco en lugares públicos ${ }^{28-35}$, es necesario promover investigaciones dirigidas a conocer el estado actual del tema y a continuar instaurando medidas que posibiliten su erradicación.

\section{Conclusiones}

Este estudio muestra que las mujeres presentan tasas de prevalencia mayores que los hombres, con un consumo medio de 1-10 cigarrillos al día entre los fumadores/as participantes en el estudio (79.3\%) y con una motivación escasa para dejar de fumar $(37.9 \%$ muestran baja motivación) y una dependencia moderada, que correlaciona con los valores obtenidos en las cooximetrías y se representa discretamente mayor en hombres, aunque la diferencia no resulta significativa con respecto a las mujeres,

Los futuros podólogos/as y enfermeros/as tienen los mismos motivos para fumar, aunque se observan diferencias significativas entre mujeres y hombres; es decir, las mujeres encuentran más motivos por los que seguir fumando. Se observan más motivos para fumar cuanto menor es la edad. El motivo "Fumo más cuando estoy preocupado por algo" es el que obtiene mayor puntuación.

En cuanto a la dependencia psicológica, social y gestual, se observa dependencia moderada en mujeres y leve en hombres (aunque la diferencia no es estadísticamente significativa), siendo menor en el alumnado de Podología que en el de Enfermería.

\section{Aplicabilidad}

Un hallazgo interesante es que los datos obtenidos en el test de Fageström son los únicos que no correlacionan con los obtenidos en el resto de los cuestionarios utilizados en dicho estudio, lo que sugiere que la dependencia a la nicotina no parece ser la causa del mantenimiento del hábito tabáquico y sugiere como más potente la dependencia psicológica, social y gestual.

\section{Bibliografía}

1. World Health Organization. Regional Office for Europe. First WHO European recommendations on treatment of tobacco dependence. Evidence based core recommendations for health care systems in Europe. WHO. Regional Office for Europe. Denmark, 2001

2. Bandura A. Teoría del aprendizaje social. Espasa universitaria. Madrid, 1984.

3. Pérez Trulle A, Herrero I, Clemente ML, Escosa L. Abordaje de la prevención y el tratamiento del tabaquismo: ¿a quién, cuándo y cómo realizar la deshabituación tabáquica? Arch. Bronconeumología 2004; 40 (supl. 3): 63-73.

4. Xunta de Galicia. Consellería de Sanidade. Dirección Xeral de Saúde Pública. Guía de atención al tabaquismo en atención primaria. 2008

5. Gil López E, Robledo de Dios T, Rubio Colavida JM, Bris Coello MR, Espiga López I, Sáiz Martínez-Acitores I. Prevalencia del consumo de tabaco en los profesionales sanitarios del INSALUD 1998, España. Prev Tab 2000; 2 (1): 22-31

6. Gutierrez García JJ, Velasco Soria A, Lozano Polo A, Usera Clavero ML. Estudio sobre tabaquismo en profesionales sanitarios de atención primaria de salud de la Región de Murcia. Consejería de Sanidad y Política Social, 2011. Serie Informes: 60.

7. Doll R, Peto R, Boreham J, Sutherland I. Mortality in relation to smoking: 50 years' observations on male British doctors. BMJ 2004; 328 (7455): 1519.

8. Jiménez Ruiz, Barrueco Ferero; Solano Reina; Torrecilla Garcia; Dominguez Grandal; Diaz-Maroto; Alonso Moreno; De la Cruz Amoros y Abengozar Muela. Recomendaciones en el Abordaje diagnóstico y terapéutica del tabaquismo. Documento de Consenso. Arch. Bronconeumologia 2003; 39 (1):35-41.

9. Jiménez Ruiz; Camarelles Guillen; Granda Orive; Diaz -Maroto Muñoz. Protocolo de intervención en fumadores. Edita EMISA; 2002

10. Chamorro García, L. Guia clínica sobre la dependencia a nicotina: SEMERGEN 2002; 28 (9): 484-8. 
11. Hernández García I, Sáenz González MC, González Celador R. Mortalidad atribuible al consumo de tabaco en España en el año 2006. Anales Sis. San Navarra 2010; 33 (1): 23-33.

12. Fagerström KO. Measuring degree of physical dependence to tobacco with reference to individualization of treatment. Addictive behavior 1978; 3: 235-241.

13. Corbalán Carrillo G, Sierra Manzano JM, Marión Navasa G, Carré Catasús M. Consumo de tabaco y dependencia de la nicotina. Rev. Rol de Enfermería n²24, abril 1997; 224: 14-16

14. Barrueco M, Aldo-Mateo Torriachi, Hernández Redero J, Sánchez Barba M, Torrecilla M, Alaejos I, et al. Fageström tolerance questionnaire \&heavy smoking índex. ¿Cúal de los dos test es suficiente para determinar la dependencia en la clínica diaria? Prev Tab 2013;15(1):13-20

15. Martin, V, Fernández, D, Ordoñez, C, Molina, A, Fernández, M. E, y de Luís, J. M. (2008). Valoración con tres métodos diferentes la prevalencia de consumo de tabaco en estudiantes de primer curso de ciencias de la salud de la universidad de León en 2006. Revista Española de Salud Pública, 82, 221-229.

16. Guía para a abordaxe do tabaquismo en grupo; manual de sesións: plan de atención ao tabaquismo en Galicia [Internet]. Santiago de Compostela: Xunta de Galicia, Servicio Galego de Saúde; 2008.

17. Treating tobacco use and dependence: 2008 update [Internet]. Clinical Practice Guideline. [Washington DC]: Departament of Health and Human Services. Public Health Service; 2008 May.

18. Russell MA, Wilson C, Taylor C, Baker CD. Effect of general practitioner's advice against smoking. Br Med J 1979; 2: 231 - 5.

19 Convenio Marco de la OMS para el control del tabaco. Ginebra: OMS; 2003.

20. Ley $28 / 2005$, de 26 de Diciembre, de medidas sanitarias frente al tabaquismo y reguladora de la venta, el suministro, el consumo y la publicidad de los productos del tabaco. BOE [Internet]2005 Dic 27; ( $\left.n^{\circ} 309\right)$

21. Alberdi-Erice MJ, Huizi-Egilegor X, Barandiarán-Lasa M, ZupiriaGorostidi X, Uranga-Iturrioz MJ. Evolución de los hábitos de consumo de tabaco y alcohol en estudiantes de enfermería. Enfermería clínica. 2007; 17(2): 63-70.

22. INE base [Internet]: Madrid: Instituto Nacional de Estadïstica, 2014. Población de 16 y más años por edad, sexo y consumo de tabaco [tabla]. Disponible en: http://www.ine.es/dynt3/inebase/index.htm?type=pcaxis\&path $=/ \mathrm{t} 15 / \mathrm{p} 419 / \mathrm{p} 02 / \mathrm{a} 2003 /$ \&file=pcaxis.

23. Míguez MC, Becoña E. Consumo de tabaco y alcohol en la población escolar de Galicia. Revista española de drogodependencias. 2066; 31(1): 46-56. Disponible en: http://www.aesed.com/descargas/revistas/v31n1_4.pdf

24. Agencia de Evaluación de Tecnologías Sanitarias (AETS). Instituto de Salud Carlos III. Ministerio de Sanidad y Consumo. Evaluación de la eficacia, efectividad y coste-efectividad de los distintos abordajes terapéuticos para dejar de fumar. AETS, Madrid, Septiembre 2003.

25. Prochaska J, Di Clemente C. Stages and process of self-change of smoking: towards an integrative model of change. J Consult Clin Psychol 1983; 51:390-395.

26. Programa Galego de Promoción da Vida sen Tabaco. Informe 1993-1998. Santiago de Compostela: Consellería de Sanidade e Servicios Sociais, Dirección Xeral de Saúde Pública; 2000. Serie II: Sección Tabaco. Informe 2.

27. O consumo de tabaco nos profesionais sanitarios de Galicia, ano 2006. Boletín Epidemiolóxico de Galicia (BEG), 2006; 39

28. Catalina Romero $C$, et al. Prevalencia de consumo de tabaco en población trabajadora tras la entrada en vigor de la Ley 42/2010. Revista española de salud pública. 2012; 86(2): 177-188. Disponible en: http://scielo.isciii.es/pdf/resp/v86n2/06_original5.pdf

29. Catalina Romero C, Gelpi Médez JA, Cortés Arcas MV, Martín Barallat J. Evolución en España del consumo de tabaco en población trabajadora desde la entrada en vigor de la Ley 28/2005 de medidas sanitarias frente al tabaquismo. Rev Esp Salud Pública. 2010; 84: 223-227. Disponible en:

http://www.mscbs.gob.es/biblioPublic/publicaciones/recurso s_propios/resp/revista_cdrom/vol84/vol84_2/RS842C_223.pdf

30. Gallego Alonso-Colmenero MM, De Miguel Díez J, Ávillar Lvarez F, Pérez De Oteyza C. Tobacco consumption in hospitalized patients before and after the anti-tobacco law (28/2005). [Consumo de tabaco en pacientes hospitalizados antes y después de la Ley antitabaco (28/2005)]. Rev. Clin. Esp. 2010; 210(5): 216220

31. González-Torrente S, Bennasar-Veny M, Pericás-Beltrán J, PedroGómez JE, Aguiló-Pons A, Lluc Bauzá-Amengual M. Hábito tabáquico entre los estudiantes de enfermería y fisioterapia de la Universitat de les Illes Balears: opinión ante la regulación del consumo en lugares públicos. Enfermería clínica. 2008; 18(5): 245-252.

32. Lushchenkova O, Fernández E, López MJ, Fu M, Martínez-Sánchez JM, Nebot M, et al. Secondhand smoke exposure in Spanish adult non-smokers following the introduction of an anti-smoking law. [Exposición al humo ambiental de tabaco en población adulta no fumadora en España tras la ley de medidas sanitarias frente al tabaquismo]. Rev. Esp. Cardiol. 2008; 61(7): 687-694

33. Manzanares-Laya S, López MJ, Sánchez-Martínez F, Fernández E, Nebot M. Impact of the $28 / 2005$ Spanish smoking law on second-hand smoke exposure in Barcelona. [Impacto de la Ley $28 / 2005$ de medidas sanitarias frente al tabaquismo en la prevalencia de la exposición al humo ambiental del tabaco en Barcelona]. Gaceta Sanitaria 2011;25(6): 495-500

34. Martin V, Fernández D, Ordóñez C, Molina AJ, Fernández E, Luis JM de. Valoración con tres métodos diferentes de la prevalencia de consumo de tabaco en estudiantes de primer curso de ciencias de la salud de la Universidad de León en 2006. Revista española de salud pública. 2008; 82(2): 221-229. Disponible en: http://recyt.fecyt.es/index.php/RESP/article/view/899

35. Míguez Varela MC, Becoña Iglesias E. El consumo de tabaco en estudiantes de psicología a lo largo de 10 años. Psicothema. 2009; 21(4): 573-578

Disponible en: http://www.psicothema.com/pdf/3673.pdf

36. Plan Nacional sobre Drogas [Internet]. Madrid: Ministerio de Sanidad, Servicios Sociales e Igualdad; 2012. Encuesta Estatal sobre Uso de Drogas en Estudiantes de Enseñanzas Secundarias (ESTUDES), 1994-2010. Disponible en: http://www.pnsd.msc.es/Categoria2/observa/pdf/ESTUDES_2 010.pdf

37. Romero CC, Gutierrez JCS, Aguado LQ, Arcas MVC, Blazquez JAP, Mendez JAG, et al. Prevalence of tobacco consumption amongworking population after the law 42/2010, Spain. [Prevalenciade consumo de tabaco en poblacion trabajadora tras la entrada en vigor de laley 42/2010]. Rev. Esp. Salud Publica 2012; 86(2): 177188

38. Sánchez Beiza L, Martin-Carrillo Dominguez P, Gil Serrano MP. Detección y características del consumo de drogas legales en jóvenes de 15 a 25 años. Aten Primaria 1993; 11: 350-352

39. Ratificación del Convenio Marco de la OMS para el control del tabaco, hecho en Ginebra el 21 de mayo de 2003. BOE [Internet]2005 Feb 10 ( $\left.\mathrm{N}^{\circ} 35\right)$.

40. Ley $42 / 2010$, de 30 de Diciembre, de Medidas Sanitarias frente al tabaquismo y reguladora de la venta, el suministro, el consumo y la publicidad de los productos del tabaco. BOE [Internet] 2010Dic $31\left(N^{\circ} 318\right)$

41. 0 tabaquismo entre os profesionais sanitarios de Galicia. Boletín Epidemiolóxico de Galicia (BEG). 2001; (4). 
Anexo:

Tablas

\begin{tabular}{|c|c|c|c|c|}
\hline Variables & $\mathbf{n}$ & $\%$ & 95 \% I.C. & Media \pm DT \\
\hline Edad & 81 & & & $21.8 \pm 5.0$ \\
\hline \multicolumn{5}{|l|}{ Sexo } \\
\hline Mujer & 69 & $84.1 \%$ & & \\
\hline Hombre & 13 & $15.9 \%$ & & \\
\hline \multicolumn{5}{|l|}{ Centro académico } \\
\hline Enfermería (1er. curso) & 50 & $61.0 \%$ & & \\
\hline Podología (3er. curso) & 32 & $39.0 \%$ & & \\
\hline \multicolumn{5}{|l|}{ Hábito tabáquico } \\
\hline No fumador & 46 & $56.1 \%$ & & \\
\hline Fumador actual & 31 & $37.8 \%$ & & \\
\hline Exfumador & 5 & $6.1 \%$ & & \\
\hline \multicolumn{5}{|l|}{ Cigarillos/día (fumadores) } \\
\hline $1-10$ & 23 & $79.3 \%$ & & \\
\hline $11-20$ & 6 & $20.7 \%$ & & \\
\hline
\end{tabular}

Tabla 1. Características de la muestra estudiada según diferentes variables

\begin{tabular}{|l|c|c|c|c|c|}
\hline Variable & $\mathbf{n}$ & Media \pm DT & Mediana & Mínimo & Máximo \\
\hline p.p.m. & 78 & $3.08 \pm 4.31$ & 2 & 0 & 24 \\
\hline Fco hb & 78 & $1.49 \pm 0.46$ & 1.46 & 0 & 2.38 \\
\hline Carboxi Hb & 78 & $1.14 \pm 0.71$ & 1 & 0 & 4.5 \\
\hline
\end{tabular}

Tabla 2. Hallazgos de la Cooximetría en la totalidad de la muestra estudiada

\begin{tabular}{|c|c|c|c|c|}
\hline & $\begin{array}{l}\text { Nunca Fumador } \\
(\mathrm{n}=44) \\
\text { Media } \pm \text { DT }\end{array}$ & $\begin{array}{l}\text { Ex Fumador } \\
(n=4) \\
\text { Media } \pm D T\end{array}$ & $\begin{array}{l}\text { Fumador } \\
(n=30) \\
\text { Media } \pm D T\end{array}$ & p \\
\hline p.p.m. & $1.44 \pm 0.86$ & $1.45 \pm 0.64$ & $5.71 \pm 6.04$ & 0.000 \\
\hline Fco hb & $1.32 \pm 0.42$ & $1.46 \pm 0.0$ & $1.73 \pm 0.43$ & 0.000 \\
\hline Carboxi Hb & $0.85 \pm 0.30$ & $0.95 \pm 0.10$ & $1.59 \pm 0.94$ & 0.000 \\
\hline
\end{tabular}

Tabla 3. Hallazgos de la Cooximetría según el hábito tabáquico declarado 


\begin{tabular}{|c|c|c|}
\hline Cuestionario Richmond & $\mathbf{n}$ & $\%$ \\
\hline \multicolumn{3}{|c|}{ 1. ¿Le gustaría dejar de fumar si pudiera hacerlo fácilmente? } \\
\hline No & 4 & 0.138 \\
\hline Sí & 25 & 0.862 \\
\hline \multicolumn{3}{|c|}{ 2. ¿Cuánto interés tiene usted en dejarlo? } \\
\hline Ninguno & 3 & 0.103 \\
\hline Algo & 12 & 0.414 \\
\hline Bastante & 9 & 0.31 \\
\hline Mucho & 5 & 0.172 \\
\hline \multicolumn{3}{|c|}{ 3. ¿Intentará usted dejar de fumar en las próximas dos semanas? } \\
\hline Definitivamente no & 18 & 0.621 \\
\hline Quizá & 5 & 0.172 \\
\hline Sí & 5 & 0.172 \\
\hline Definitivamente sí & 1 & 0.034 \\
\hline \multicolumn{3}{|c|}{ 4. ¿Cúal es la probabilidad de que usted sea un no fumador dentro de los próximos 6 meses? } \\
\hline Muy baja & 4 & 0.138 \\
\hline Baja & 11 & 0.379 \\
\hline Alta & 10 & 0.345 \\
\hline Muy alta & 4 & 0.138 \\
\hline \multicolumn{3}{|l|}{ Categorías de Richmond } \\
\hline Débil (de 0 a 5) & 18 & 62.1 \\
\hline Moderada (de 6 a 8) & 9 & 31.0 \\
\hline Fuerte & 2 & 6.9 \\
\hline
\end{tabular}

Tabla 4. Descripción de los fumadores según su motivación para dejar de fumar del cuestionario de Richmond

\begin{tabular}{|l|c|c|c|c|}
\hline & B & Error & $\begin{array}{c}\text { Coeficiente estandarizado } \\
(\boldsymbol{\beta})\end{array}$ & $\mathbf{p}$ \\
\hline Género & 1.23 & 1.24 & 0.21 & 0.33 \\
\hline Edad & -0.15 & 0.13 & -0.025 & 0.91 \\
\hline CarboxiHB & 0.34 & 0.54 & 0.14 & 0.53 \\
\hline
\end{tabular}
$\mathbf{R}^{2}=\mathbf{0 . 0 6 4}$

Tabla 5. Modelo de regresión lineal múltiple para predecir puntuación de Richmond ajustando por diferentes covariables 


\begin{tabular}{|c|c|c|}
\hline Cuestionario Fagerström & $\mathbf{n}$ & $\%$ \\
\hline \multicolumn{3}{|c|}{ 1. ¿Cuántos cigarros fuma al día? } \\
\hline 10 o menos & 25 & 0,862 \\
\hline 11 a 20 & 4 & 0,138 \\
\hline 21 a 30 & 0 & - \\
\hline 31 o más & 0 & - \\
\hline \multicolumn{3}{|c|}{ 2. ¿En cuánto tiempo después de despertarse fuma el primer cigarro del día? } \\
\hline En 5 minutos o menos & 22 & 0,759 \\
\hline Entre $6-30$ minutos & 2 & 0,069 \\
\hline Entre 30-60 minutos & 3 & 0,103 \\
\hline Después de 60 minutos & 2 & 0,069 \\
\hline \multicolumn{3}{|c|}{ 3. Después de fumar el primer cigarro del día, ¿fuma rápidamente algunos más? } \\
\hline Sí & 25 & 0,862 \\
\hline No & 4 & 0,138 \\
\hline \multicolumn{3}{|c|}{ 4. ¿Tiene dificultades para abstenerse de fumar en lugares donde está prohibido hacerlo? } \\
\hline Sí & 23 & 0,793 \\
\hline No & 6 & 0,207 \\
\hline \multicolumn{3}{|c|}{5 . ¿Fuma cuando no se encuentra bien o cuando está enfermo en cama? } \\
\hline Sí & 26 & 0,897 \\
\hline No & 3 & 0,103 \\
\hline \multicolumn{3}{|c|}{ 6. De todos los cigarros que fuma a lo largo del día, ¿cúal es el que más necesita? } \\
\hline El primero de la mañana & 26 & 0,897 \\
\hline Cualquiera otro & 3 & 0,103 \\
\hline
\end{tabular}

\section{Categorías de Fagerström}

No dependencia a la nicotina (0 puntos)

Dependencia a la nicotina (1 o más puntos)

Tabla 6. Descripción de los fumadores según su dependencia a la nicotina (Test de Fagerström)

\begin{tabular}{|l|c|c|c|c|c|c|}
\hline & B & Error & p & O.R. & \multicolumn{2}{|c|}{$95 \%$ I.C. } \\
\hline Sexo (Hombre vs. Mujer) & 0.23 & 1.01 & 0.82 & 1.26 & 0.17 & 9.1 \\
\hline CarboxiHB & 1.05 & 0.63 & 0.09 & 2.87 & 0.84 & 9.77 \\
\hline
\end{tabular}

Tabla 7. Modelo de regresión logística para predecir dependencia a la nicotina ajustando por sexo y niveles de carboxiHb 


\begin{tabular}{|c|c|c|c|c|}
\hline Pregunta & $\begin{array}{c}0 \\
\mathrm{n}(\%)\end{array}$ & $\begin{array}{c}1 \\
\mathrm{n}(\%)\end{array}$ & $\begin{array}{c}2 \\
\mathrm{n}(\%)\end{array}$ & $\begin{array}{c}3 \\
\mathrm{n}(\%)\end{array}$ \\
\hline $\begin{array}{l}\text { 1. Siento un gran deseo de fumar cuando tengo que } \\
\text { parar cualquier actividad por un momento }\end{array}$ & $8(27,6 \%)$ & $15(51,7 \%)$ & $3(10,3 \%)$ & $3(10,3 \%)$ \\
\hline $\begin{array}{l}\text { 2. Enciendo un cigarro sin darme cuenta de que tengo } \\
\text { otro encendido en el cenicero }\end{array}$ & $29(100 \%)$ & 53 & & \\
\hline $\begin{array}{l}\text { 3. Me gusta fumar, sobre todo cuando descanso } \\
\text { tranquilamente }\end{array}$ & $9(31 \%)$ & $4(13,8 \%)$ & $9(31 \%)$ & $7(24,1 \%)$ \\
\hline 4. Obtengo un gran placer de fumar, sea cuando sea & $10(34,5 \%)$ & $11(37,9 \%)$ & $6(20,7 \%)$ & $2(6,9 \%)$ \\
\hline $\begin{array}{l}\text { 5. Tener un cigarro entre los dedos es parte del placer } \\
\text { de fumar }\end{array}$ & $12(41,4 \%)$ & $9(31 \%)$ & $6(20,7 \%)$ & $2(6,9 \%)$ \\
\hline $\begin{array}{l}\text { 6. Pienso que mejora mi aspecto con un cigarro en las } \\
\text { manos }\end{array}$ & $28(96,6 \%)$ & $1(3,4 \%)$ & & \\
\hline 7. Fumo más cuando estoy preocupado por algo & $5(17,2 \%)$ & $4(13,8 \%)$ & $8(27,6 \%)$ & $12(41,4 \%)$ \\
\hline 8. Me siento más estimulado y alerta cuando fumo & $17(58,6 \%)$ & $8(27,6 \%)$ & $2(6,9 \%)$ & $2(6,9 \%)$ \\
\hline 9. Fumo automáticamente a pesar de estar atento & $16(55,2 \%)$ & $11(37,9 \%)$ & $2(6,9 \%)$ & \\
\hline 10. Fumo para tener algo que hacer con las manos & $16(55,2 \%)$ & $9(31 \%)$ & $2(6,9 \%)$ & $2(6,9 \%)$ \\
\hline $\begin{array}{l}\text { 11. Cuando me quedo sin cigarros me resulta casi } \\
\text { insoportable hasta que vuelvo a tenerlos }\end{array}$ & $13(44,8 \%)$ & $13(44,8 \%)$ & $2(6,9 \%)$ & $1(3,4 \%)$ \\
\hline 12. Cuando me siento infeliz fumo más & $8(27,6 \%)$ & $11(37,9 \%)$ & $7(24,1 \%)$ & $3(10,3 \%)$ \\
\hline 13. Fumar me ayuda a aguantar cuando estoy cansado & $24(82,8 \%)$ & $4(13,8 \%)$ & $1(3,4 \%)$ & \\
\hline 14. Me resulta difícil estar una hora sin fumar & $25(86,2 \%)$ & $4(13,8 \%)$ & & \\
\hline $\begin{array}{l}\text { 15. Me encuentro a mí mismo fumando sin recordar } \\
\text { haber encendido el cigarro }\end{array}$ & $28(96,6 \%)$ & $1(3,4 \%)$ & & \\
\hline $\begin{array}{l}\text { 16. Cuando estoy cómodo y relajado es cuando más } \\
\text { deseo fumar }\end{array}$ & $10(34,5 \%)$ & $9(31 \%)$ & $8(27,6 \%)$ & $2(6,9 \%)$ \\
\hline 17. Fumar me ayuda a pensar y concentrarme & $19(65,5 \%)$ & $7(24,1 \%)$ & $1(3,4 \%)$ & $2(6,9 \%)$ \\
\hline $\begin{array}{l}\text { 18. Tengo muchas ganas de fumar cuando ya llevo un } \\
\text { rato sin fumar }\end{array}$ & $7(24,1 \%)$ & $14(48,3 \%)$ & $6(20,7 \%)$ & $2(6,9 \%)$ \\
\hline 19. Me siento más maduro y sofisticado cuando fumo & $27(93,1 \%)$ & $1(3,4 \%)$ & $1(3,4 \%)$ & \\
\hline $\begin{array}{l}\text { 20. Cuando no estoy fumando soy muy consciente de } \\
\text { ello }\end{array}$ & $18(62,1 \%)$ & $10(34,5 \%)$ & $1(3,4 \%)$ & \\
\hline $\begin{array}{l}\text { 21. Me resultaría muy difícil estar una semana sin } \\
\text { fumar }\end{array}$ & $8(27,6 \%)$ & $7(24,1 \%)$ & $8(27,6 \%)$ & $6(20,7 \%)$ \\
\hline 22. Fumo para tener algo que llevarme a la boca & $40(69 \%)$ & $5(17,2 \%)$ & $4(13,8 \%)$ & \\
\hline $\begin{array}{l}\text { 23. Me siento más atractivo frente a personas del sexo } \\
\text { contrario cuando fumo }\end{array}$ & $26(89,7 \%)$ & $2(6,9 \%)$ & $1(3,4 \%)$ & \\
\hline 24. Cuando estoy enfadado enciendo un cigarro & $4(13,8 \%)$ & $6(20,7 \%)$ & $10(34,5 \%)$ & $9(31 \%)$ \\
\hline
\end{tabular}

0 : No le ocurre en absoluto; 1: Le sucede un poco; 2: Le sucede bastante; 3: Le sucede mucho 


\begin{tabular}{|c|c|c|c|c|c|c|c|c|c|c|c|}
\hline \multirow{2}{*}{ Ítems } & \multicolumn{11}{|c|}{ Escala } \\
\hline & 0 & 1 & 2 & 3 & 4 & 5 & 6 & 7 & 8 & 9 & 10 \\
\hline 1.Si me sintiera ansioso & 0.103 & 0.069 & & 0.069 & 0.034 & 0.069 & 0.034 & 0.069 & 0.103 & 0.172 & $27.6 \%$ \\
\hline $\begin{array}{l}\text { 2. Si quisiera sentarme cómoda- } \\
\text { mente y disfrutar un cigarrillo }\end{array}$ & 0.138 & & 0.034 & 0.069 & 0.034 & 0.138 & 0.034 & 0.241 & 0.069 & 0.207 & 0.034 \\
\hline $\begin{array}{l}\text { 3. Si termino una comida o un } \\
\text { tentempié }\end{array}$ & 0.138 & & 0.034 & 0.034 & 0.172 & 0.034 & 0.034 & & 0.069 & 0.207 & $27.6 \%$ \\
\hline 4. Si me sintiera nervioso & 0.103 & 0.103 & & 0.034 & 0.034 & 0.069 & 0.034 & 0.034 & 0.103 & 0.207 & $27.6 \%$ \\
\hline 5. Si quisiera sentirme más atracti & $\infty .759$ & 0.069 & 0.034 & & 0.034 & 0.034 & & 0.034 & & & 0.034 \\
\hline 6. Si quisiera relajarme & 0.172 & & 0.034 & 0.034 & 0.103 & 0.138 & 0.172 & 0.207 & 0.138 & & \\
\hline $\begin{array}{l}\text { 7. Si pensara que fumar es parte } \\
\text { de mi auto-imagen }\end{array}$ & 0.759 & 0.069 & 0.034 & 0.034 & 0.034 & & & & 0.034 & & 0.034 \\
\hline 8. Si me sintiera tenso & 0.103 & 0.069 & 0.034 & 0.034 & 0.034 & 0.069 & 0.069 & 0.034 & $27.6 \%$ & 0.138 & 0.138 \\
\hline $\begin{array}{l}\text { 9. Si estuviera bebiendo una } \\
\text { bebida alcohólica }\end{array}$ & 0.103 & 0.034 & & 0.034 & 0.034 & 0.069 & & 0.034 & 0.138 & 0.207 & 0.345 \\
\hline 10. Si viera a otros fumando & 0.207 & & & & & 0.138 & 0.034 & 0.207 & 0.172 & 0.172 & 0.069 \\
\hline $\begin{array}{l}\text { 11. Si alguien me ofreciera un } \\
\text { cigarrillo }\end{array}$ & 0.138 & 0.069 & & & 0.034 & 0.172 & 0.138 & 0.034 & $27.6 \%$ & 0.103 & 0.034 \\
\hline $\begin{array}{l}\text { 12. Si quisiera evitar comer algo } \\
\text { dulce }\end{array}$ & 0.448 & 0.034 & 0.034 & 0.103 & 0.069 & 0.103 & 0.034 & 0.069 & 0.069 & & 0.034 \\
\hline $\begin{array}{l}\text { 13. Si quisiera sentirme más } \\
\text { maduro y sofisticado }\end{array}$ & 0.759 & 0.069 & 0.103 & & & & & 0.034 & & & 0.034 \\
\hline $\begin{array}{l}\text { 14. Si pensara que esto me } \\
\text { ayudaría a mantener mi peso }\end{array}$ & 0.517 & 0.069 & 0.069 & 0.034 & & 0.172 & & & 0.069 & & 0.069 \\
\hline
\end{tabular}

0 mínimo, 10 máximo

Tabla 9. Resultados del cuestionario de Baer \& Lichtenstein

\begin{tabular}{|l|c|c|c|c|}
\hline & B & Error & $\begin{array}{c}\text { Coeficiente estandarizado } \\
(\boldsymbol{\beta})\end{array}$ & $\mathbf{p}$ \\
\hline Género & -33.35 & 10.76 & -0.55 & 0.005 \\
\hline Edad & -0.69 & 1.15 & -0.11 & 0.55 \\
\hline CarboxiHB & 4.74 & 4.66 & 0.18 & 0.32 \\
\hline
\end{tabular}

Tabla 10. Modelo de regresión lineal múltiple de la puntuación de Baer \& Lichtenstein ajustando por género, edad y valores de carboxiHb 


\begin{tabular}{|c|c|c|}
\hline Cuestionario Glover-Nilsson & $\mathbf{n}$ & $\%$ \\
\hline \multicolumn{3}{|c|}{ 1. Mi hábito de fumar es muy importante para mí } \\
\hline $0:$ Nada & 6 & 0,207 \\
\hline 1: Algo & 12 & 0,414 \\
\hline 2: Moderadamente & 9 & 0,31 \\
\hline 3: Mucho & 1 & 0,034 \\
\hline 4: Muchísimo & 1 & 0,034 \\
\hline \multicolumn{3}{|c|}{ 2. Juego y manipulo el cigarro como parte del ritual del hábito de fumar } \\
\hline $0:$ Nada & 15 & 0,517 \\
\hline 1: Algo & 10 & 0,345 \\
\hline 2: Moderadamente & 2 & 0,069 \\
\hline 3: Mucho & 1 & 0,034 \\
\hline 4: Muchísimo & 1 & 0,034 \\
\hline \multicolumn{3}{|c|}{ 3. Suelo ponerme algo en la boca para evitar fumar } \\
\hline $0:$ Nada & 22 & 0,759 \\
\hline 1: Algo & 6 & 0,207 \\
\hline 2: Moderadamente & 1 & 0,034 \\
\hline \multicolumn{3}{|l|}{ 3: Mucho } \\
\hline \multicolumn{3}{|l|}{ 4: Muchísimo } \\
\hline \multicolumn{3}{|c|}{ 4. Cuando no tengo tabaco me resulta muy difícil concentrarme y realizar cualquier tarea } \\
\hline $0:$ Nada & 14 & 0,483 \\
\hline 1: Algo & 10 & 0,345 \\
\hline 2: Moderadamente & 4 & 0,138 \\
\hline 3: Mucho & 1 & 0,034 \\
\hline 4: Muchísimo & & \\
\hline
\end{tabular}

Tabla 11. Cuestionario de Glover-Nilsson 


\begin{tabular}{|c|c|c|c|c|c|}
\hline Cuestionario Glover Nilsson & $\begin{array}{c}0 \\
\mathrm{n}(\%)\end{array}$ & $\begin{array}{c}1 \\
\mathrm{n}(\%)\end{array}$ & $\begin{array}{c}2 \\
\mathrm{n}(\%)\end{array}$ & $\begin{array}{c}3 \\
n(\%)\end{array}$ & $\begin{array}{c}4 \\
\mathrm{n}(\%)\end{array}$ \\
\hline 1. Mi hábito de fumar es muy importante para mí & $6(20,7 \%)$ & $12(41,4 \%)($ & $9(31 \%)$ & $1(3,4 \%)$ & $1(3,4 \%)$ \\
\hline $\begin{array}{l}\text { 2. Juego y manipulo el cigarro como parte del } \\
\text { ritual del hábito de fumar }\end{array}$ & $15(51,7 \%)$ & $10(34,5 \%)$ & $2(6,9 \%)$ & $1(3,4 \%)$ & $1(3,4 \%)$ \\
\hline $\begin{array}{l}\text { 3. Suelo ponerme algo en la boca para evitar } \\
\text { fumar }\end{array}$ & $22(75,9 \%)$ & $6(20,7 \%)$ & $1(3,4 \%)$ & & \\
\hline $\begin{array}{l}\text { 4. Cuando no tengo tabaco, me resulta muy } \\
\text { difícil concentrarme y realizar cualquier tarea }\end{array}$ & $14(48,3 \%)$ & $10(34,5 \%)$ & $4(13,8 \%)$ & $1(3,4 \%)$ & \\
\hline $\begin{array}{l}\text { 5. Cuando me encuentro en algún lugar donde } \\
\text { está prohibido fumar, juego con un cigarro o con } \\
\text { el paquete de tabaco }\end{array}$ & $24(82,8 \%)$ & $1(3,4 \%)$ & $4(13,8 \%)$ & & \\
\hline $\begin{array}{l}\text { 6. Me recompenso con un cigarro tras finalizar } \\
\text { una tarea }\end{array}$ & $6(20,7 \%)$ & $5(17,2 \%)$ & $10(34,5 \%)$ & $6(20,7 \%)$ & $2(6,9 \%)$ \\
\hline $\begin{array}{l}\text { 7. Algunos lugares o circunstancias me incitan a } \\
\text { fumar (sofá preferido, habitación, coche, } \\
\text { bebida..) }\end{array}$ & $1(3,4 \%)$ & $5(17,2 \%)$ & $5(17,2 \%)$ & $7(24,1 \%)$ & $11(37,9 \%)$ \\
\hline $\begin{array}{l}\text { 8. A menudo me coloco en los labios un cigarro } \\
\text { sin encender u otros objetos (palillos, bolígrafos) } \\
\text { y los chupo para aliviar el estrés, ansiedad, } \\
\text { frustración... }\end{array}$ & $23(79,3 \%)$ & $2(6,9 \%)$ & $4(13,8 \%)$ & & \\
\hline $\begin{array}{l}\text { 9. Me encuentro habitualmente encendiendo un } \\
\text { cigarro por rutina sin desearlo realmente }\end{array}$ & $14(48,3 \%)$ & $10(34,5 \%)$ & $4(13,8 \%)$ & $1(3,4 \%)$ & \\
\hline $\begin{array}{l}\text { 10. Parte de mi placer de fumar proviene del } \\
\text { ritual que supone encender un cigarrillo }\end{array}$ & $17(58,6 \%)$ & $7(24,1 \%)$ & $4(13,8 \%)$ & $1(3,4 \%)$ & \\
\hline $\begin{array}{l}\text { 11. Cuando estoy solo en un restaurante, parada } \\
\text { de autobús, reunión, fiesta..., me siento más } \\
\text { seguro con un cigarro en las manos }\end{array}$ & $10(34,5 \%)$ & $3(10,3 \%)$ & $8(27,6 \%)$ & $5(17,2 \%)$ & $3(10,3 \%)$ \\
\hline
\end{tabular}

Puntuación total Glover Nilsson 


\begin{tabular}{|l|c|c|c|c|}
\hline \multirow{2}{*}{ Cuestionarios } & $\begin{array}{c}\text { Cuestionario de } \\
\text { Richmond }\end{array}$ & $\begin{array}{c}\text { Cuestionario de } \\
\text { Fageström }\end{array}$ & $\begin{array}{c}\text { Cuestionario de } \\
\text { Russell }\end{array}$ & $\begin{array}{c}\text { Cuestionario de } \\
\text { Glover-Nilsson }\end{array}$ \\
\cline { 2 - 5 } & $\mathrm{r} ; \mathrm{p}) ; \mathrm{n}$ & $\mathrm{r} ; \mathrm{p}) ; \mathrm{n}$ & $\mathrm{r} ;(\mathrm{p}) ; \mathrm{n}$ & $\mathrm{r} ;(\mathrm{p}) ; \mathrm{n}$ \\
\hline Richmond & & $-0.19 ;(0.33) ; 29$ & $-0.42 ;(0.03) \star ; 29$ & \\
\hline Fageström & & & $0.5 ;(0.06) ; 29$ & \\
\hline Baer \& Lichtenstein & $-0.59 ;(0.01) \star * ; 29$ & $0.24 ;(0.21) ; 29$ & $0.79 ;(0.00) \star * ; 29$ & $0.72 ;(0.00) ; 29$ \\
\hline Glover-Nilsson & $-0.45 ;(0.01) * ; 29$ & $0.43 ;(0.21) * ; 29$ & $0.85 ;(0.00) \star * ; 29$ & \\
\hline
\end{tabular}

* La correlación es significante al nivel 0,05 (bilateral)

** La co rrelación es significativa al nivel 0,01(bilateral)

Tabla 13. Correlación entre los diferentes test utilizados

\begin{tabular}{|l|l|l|l|l|l|l|}
\hline Cuestionarios & $\begin{array}{l}\text { Sexo } \\
\text { (media } \pm \text { sd) }\end{array}$ & $\begin{array}{l}\text { Centros } \\
\text { (media } \pm \text { sd) }\end{array}$ \\
\hline & Mujeres & Hombres & $\mathbf{p}$ & Enfermería & Podología & p \\
\hline Richmond & $4.3 \pm 2.4$ & $5.3 \pm 3.1$ & 0.38 & $3.9 \pm 2.8$ & $5.1 \pm 2.2$ & 0.23 \\
\hline Fageström & $1.13 \pm 1.9$ & $1.33 \pm 1.7$ & 0.82 & $0.93 \pm 1.26$ & $1.4 \pm 2.32$ & 0.51 \\
\hline Russell & $20.09 \pm 10.29$ & $8 \pm 6.4$ & $\mathbf{0 . 0 1}$ & $17.5 \pm 9.93$ & $17.67 \pm 11.79$ & 0.97 \\
\hline Baer \& Lichtenstein & $69.91 \pm 5.23$ & $38.33 \pm 21.53$ & $\mathbf{0 . 0 0 9}$ & $63.57 \pm 28.43$ & $63.2 \pm 27.26$ & 0.97 \\
\hline Glover-Nilsson & $12.57 \pm 7.4$ & $5.67 \pm 4.72$ & $\mathbf{0 . 0 4}$ & $12.5 \pm 7.1$ & $9.87 \pm 7.74$ & 0.35 \\
\hline
\end{tabular}

Tabla 14. Media de la puntuación de los diferentes cuestionarios según las variables sexo y centro de estudio

\begin{tabular}{|l|c|c|c|c|c|}
\hline \multirow{3}{*}{ Cuestionarios } & & \multicolumn{3}{|c|}{ Cooximetría } \\
\cline { 2 - 6 } & Edad de inicio & Edad Actual & p.p.m & CarboxiHB & FcoHB \\
\cline { 2 - 6 } & $\mathrm{r} ; \mathrm{p}) ; \mathrm{n}$ & $\mathrm{r} ; \mathrm{p}) ; \mathrm{n}$ & $\mathrm{r} ; \mathrm{p}) ; \mathrm{n}$ & $\mathrm{r} ; \mathrm{p}) ; \mathrm{n}$ & $\mathrm{r} ; \mathrm{p}) ; \mathrm{n}$ \\
\hline Richmond & $0.13 ;(0.54) ; 25$ & $0.24 ;(0.23) ; 28$ & $0.08 ;(0.67) ; 28$ & $0.03 ;(0.88) ; 28$ & $-0.2 ;(0.32) ; 28$ \\
\hline Fageström & $0.14 ;(0.52) ; 25$ & $0.06 ;(0.75) ; 28$ & $0.30 ;(0.11) ; 29$ & $0.33 ;(0.09) ; 28$ & $-0.03 ;(0.89) ; 28$ \\
\hline Russell & $-0.13 ;(0.54) ; 25$ & $-0.41 ;(0.03) ; 28$ & $0.31 ;(0.11) ; 28$ & $0.32 ;(0.09) ; 28$ & $0.06 ;(0.76) ; 28$ \\
\hline Baer \& Lichtenstein & $0.08 ;(0.69) ; 25$ & $-0.24 ;(0.22) ; 28$ & $0.15 ;(0.46) ; 28$ & $0.16 ;(0.41) ; 28$ & $0.19 ;(0.34) ; 28$ \\
\hline Glover-Nilsson & $-0.14 ;(0.52) ; 25$ & $-0.39 ;(0.04) ; 28$ & $0.19 ;(0.33) ; 28$ & $0.22 ;(0.27) ; 28$ & $0.02 ;(0.92) ; 28$ \\
\hline
\end{tabular}

Tabla 15. Correlación de diferentes covariables con los cuestionarios utilizados 